\title{
Objective classification of specific radiation damage in macromolecular X-ray crystallography
}

\author{
Charles S. Bury ${ }^{1}$, John E. McGeehan ${ }^{2}$, Ian Carmichael ${ }^{3}$, Elspeth F. Garman ${ }^{1}$ \\ ${ }^{1}$ Department of Biochemistry, University of Oxford, South Parks Road, Oxford, OX1 3QU \\ ${ }^{2}$ Biophysics Laboratories, Institute of Biomedical and Biomolecular Sciences, \\ University of Portsmouth, King Henry I Street, Portsmouth, Hampshire PO1 2DY, UK \\ ${ }^{3}$ Notre Dame Radiation Laboratory, University of Notre Dame, Notre Dame, IN 46556, USA
}

In macromolecular X-ray crystallography, radiation damage is still a major limiting factor to successful data collection from crystals held at both room and cryocooled temperatures $(100 \mathrm{~K})^{1}$. For experiments conducted at $100 \mathrm{~K}$, protein specific damage to particular amino acids has been widely reported at doses of just several MGy, before any observable decay in average diffraction intensities. When undetected, such artifacts of X-ray irradiation lead to errors in the assignment of active site geometry and protein-nucleic acid interactions, and ultimately, failure to elucidate the functional importance of residues.

$F_{o b s}(n)-F_{o b s}(1)$ Fourier difference maps are a widely utilised tool for detecting damage events with absorbed dose. However, visual interpretation of such maps is hindered by inherent subjective bias and is intractable for large structures. To address this challenge, a new computational method, RIDL, has been developed to enable objective quantification of specific damage events in macromolecular structures using difference electron density ${ }^{2,3}$. Machine learning algorithms have recently proved promising in $R I D L$ to permit identification of true damage events within noisy electron difference density maps, whilst guided by prior knowledge from model protein systems for which radiation damage behaviour has been well characterised. Ultimately, such analysis aims to make specific damage detection a more tractable component of structure validation.

1. Garman,EF (2010) Acta Cryst. D66 (4): 339-51

2. Bury,CS, et al. (2016) Acta Cryst. D72 (5): 648-657

3. Bury,CS, et al. RIDL in preparation.

Email corresponding author: charles.bury@dtc.ox.ac.uk 